\title{
Association of the Arg72Pro Polymorphism in p53 with Progression of Diabetic Nephropathy in T2DM Subjects
}

\section{Katarina Kuricova1', Lukas Pacal', Veronika Dvorakova' and Katerina Kankova ${ }^{2 *}$}

${ }^{1}$ Department of Pathophysiology, Faculty of Medicine, Masaryk University, Brno, Czech Republic

${ }^{2}$ Regional Centre for Applied Molecular Oncology, Masaryk Memorial Cancer Institute, Brno, Czech Republic

\begin{abstract}
Objective: In addition to its anticancer function, p53 (regulated by murine double minute 2 oncoprotein, MDM2) has recently been shown to control intracellular metabolic processes. It participates in the regulation of glucose, fatty and amino acid and purine metabolism, influences mitochondrial integrity and oxidative phosphorylation, insulin sensitivity, antioxidant response and autophagy. With respect to the possible impact of genetic variability in p53 and MDM2 on metabolic compensation the aim of the study was to analyse the effect of common germ line Single Nucleotide Polymorphisms (SNPs) - Arg72Pro in the TP53 and SNP309 in the MDM2 - on the progression of Diabetic Nephropathy (DN), cardiovascular morbidity and mortality and all-cause mortality in Type 2 Diabetes Mellitus (T2DM) subjects
\end{abstract}

Methods: The cross-sectional study comprised a total of 309 (a sum of 155 and 154) unrelated Caucasian diabetic patients with diabetes duration at least 10 years and variable renal function at baseline $(309$, mean age was $67.2 \pm$ 10.8 years). The stage of diabetic nephropathy was defined according to the urinary albumin excretion and glomerular filtration rate. Patients were followed-up for median 37 (20-59) months. The following end-points were considered: (a) progression of DN, (b) major cardiovascular event (non-fatal or fatal myocardial infarction or stroke, limb amputation, revascularization), (c) all-cause mortality. Genotypes were determined by PCR-based methodology. Time-to-event analysis using Kaplan-Meier curves and log-rank test was used.

Results: We found significant difference between CG+GG vs. CC genotypes of the p53 Arg72Pro SNP for DN progression ( $P=0.046$, log-rank test). Carriers of genotypes containing $G$ allele (previously associated with susceptibility to T2DM) had faster progression of DN than CC genotype carriers. We did not find any significant difference between genotypes of MDM2 SNP for any of the end-points studied.

Conclusions: Presented findings in general support the role of p53 in the pathogenesis of metabolic diseases, namely progression of hyperglycemia-related morbidity. Nevertheless, further studies are warranted to elucidate the eventual causal involvement of p53 pathway in the development of diabetic complications.

Keywords: p53; Murine double minute 2 oncoprotein; Diabetic nephropathy; Cardiovascular disease; Mortality; Single nucleotide polymorphism; Association

Abbreviations: CKD: Chronic Kidney Disease; DN: Diabetic Nephropathy; MDM2: Murine Double Minute 2 Oncoprotein; PPP: Pentose Phosphate Pathway; T2DM: Type 2 Diabetes Mellitus

\section{Introduction}

The physiological role of $\mathrm{p} 53$ protein in the prevention of cancer development through blocking cell cycle progression, regulating cellular senescence or apoptosis is well established [1]. In recent years though, the emerging role of p53 in metabolic regulation has been a topic of great interest. Not only p53 is activated by stress signals, it also seems to complexly control energy metabolism under normal conditions [2]. By regulating gene expression and other indirect means p53 participates in the regulation of glucose, fatty acid, amino acid (glutaminolysis) and purine metabolism, influences mitochondrial integrity and oxidative phosphorylation, insulin sensitivity, antioxidant response, autophagy and mammalian Target of Rapamycin (mTOR) signalling to name a few [3]. Importantly, p53 induces expression of TP53-Induced Glycolysis And Apoptosis Regulator (TIGAR) which stimulates Pentose Phosphate Pathway (PPP) with subsequent production of reduced Nicotinamide Adenine Dinucleotide Phosphate (NADPH) necessary for reduction of glutathione thus supporting an efficient antioxidant defence [4]. Activation of PPP is especially relevant in diabetes where it may have protective effect counteracting the negative consequences of hyperglycaemia. PPP can process glucose intermediates accumulating due to hyperglycaemia that activate metabolic pathways largely responsible for the development and progression of microvascular diabetic complications [5].
A host of tumour-associated mutations have been described in the TP53 gene (chromosome 17p13.1) resulting in very different activities from wild-type p53 such as either loss- or gain-of-function [6]. Apart from somatic mutations, inherited germ line common polymorphisms in the TP53 gene may also contribute to the physiological and pathophysiological functions of $\mathrm{p} 53$. One of them - a guanine to cytosine exchange in exon 4 (rs1042522) of the TP53 causing substitution of arginine to proline in the codon 72 - has been widely studied in the context of various tumours since the two variants exhibited markedly different pro-apoptotic potential [7]. Due to the previously reported positive association of $\mathrm{p} 53$ polymorphism with susceptibility to Type 2 Diabetes Mellitus (T2DM) in a large scale candidate gene association study [8] and results of experimental studies indicating that p53 expression in adipose tissue is involved in the development of insulin resistance [9] the focus broadened to non-oncological fields as well. Recently, two large studies reported association of allele Arg72 with T2DM [10] and with the degree of insulin resistance in T2DM [11]

${ }^{*}$ Corresponding author: Katerina Kankova, Masaryk University, Faculty of Medicine, Department of Pathophysiology, Kamenice 5, 62500 Brno, Czech Republic, Tel: +420-549 494 525; Fax: +420-549 494 340; E-mail: kankov@med.muni.cz

Received November 30, 2013; Accepted February 07, 2014; Published February 10,2014

Citation: Kuricova K, Pacal L, Dvorakova V, Kankova K (2014) Association of the Arg72Pro Polymorphism in p53 with Progression of Diabetic Nephropathy in T2DM Subjects. J Nephrol Ther 4: 153. doi:10.4172/2161-0959.1000153

Copyright: (c) 2014 Kuricova K, et al. This is an open-access article distributed under the terms of the Creative Commons Attribution License, which permits unrestricted use, distribution, and reproduction in any medium, provided the original author and source are credited. 
and there are also reports of its association with coronary artery disease $[12,13]$.

The activity of p53 is negatively controlled by Murine Double Minute 2 Oncoprotein (MDM2) which promotes p53 degradation and is considered as a major negative regulator of p53 [14]. The levels of MDM2 strongly affect p53 response and even small increase in MDM2 level can attenuate p53 function with possible tumour development [15]. First intron of the MDM2 gene contains second promoter with binding site for p53 [16]. This intron contains a thymine to guanine exchange at position 309 which creates SP1 transcription factor binding site and increase basal levels of MDM2 [15].

Considering increasingly appreciated role of p53 in the regulation of metabolism and its close interaction with MDM2 we were interested whether common functional Single Nucleotide Polymorphisms (SNPs) in the TP53 and MDM2 genes may affect development and progression of diabetes-related morbidity and mortality. Specifically, the aim of the study was to analyse the effect of SNPs Arg72Pro in the TP53 and SNP309 in the MDM2 on the progression of Diabetic Nephropathy (DN), cardiovascular morbidity and mortality and all-cause mortality in T2DM subjects.

\section{Material and Methods}

\section{Subjects}

The study comprised a total of 309 unrelated Caucasian T2DM patients with diabetes duration at least 10 years and variable degree of impairment of renal function ( 155 men and 154 women, mean age was $67.2 \pm 10.8$ years) from South Moravia region of Czech Republic. The baseline stage of DN was defined according to the Urinary Albumin Excretion (UAE). Our study sample consisted of: normoalbuminuric subjects (UAE $<30 \mathrm{mg} / 24 \mathrm{~h}, \mathrm{n}=27$ ), subjects with persistent microalbuminuria (UAE 30-300mg/24h, $\mathrm{n}=104$ ), with persistent proteinuria (UAE $>300 \mathrm{mg} / 24 \mathrm{~h}, \mathrm{n}=130$ ) and patients with End-Stage Renal Disease (ESRD, glomerular filtration rate $<15 \mathrm{ml} / \mathrm{min} / 1.73 \mathrm{~m}^{2}$ and/or permanent renal replacement therapy, $n=48$ ) followed up in specialized nephrology units of Brno University hospitals. Clinical characteristics of the study participants are shown in Table 1. Patients were followed-up for median 37 (IQR 20 - 59) months. The following end-points were considered: (a) progression of DN (i.e. transition from any given baseline DN stage to a more advanced stage of albuminuria or ESRD, subjects with ESRD at baseline were omitted from this analysis since no further progression was possible), (b) major cardiovascular event (i.e. non-fatal or fatal myocardial infarction or stroke, limb amputation, revascularization), (c) all-cause mortality. All patients gave their signed consent prior to their inclusion in the study. The study was performed according to the recommendations of the Declaration of Helsinki and approved by the Ethical Committee of Faculty of Medicine, Masaryk University Brno.

\section{DNA isolation and genotyping}

DNA was extracted from peripheral blood samples by the phenolchloroform method and stored at $-20^{\circ} \mathrm{C}$ until further analysis. Genotyping of TP53 SNP was performed using TaqMan ${ }^{\circledR}$ SNP Genotyping Assay (C_2403545_10) in ABI Prism ${ }^{\circledR} 7000$ Sequence Detection System with genotype discrimination performed using SDS 2.3 software (all from Applied Bio systems, Foster City, CA). MDM2 SNP was detected as previously described [17].

\section{Statistical analysis}

Comparisons between DN groups were performed by KruskalWallis ANOVA. For both SNPs, Hardy-Weinberg Equilibrium (HWE) was tested by chi-square test. Time-to-event analysis using KaplanMeier curves and log-rank test was used. For all standard analyses Statistica for Windows (Statsoft inc., Tulsa, OK, USA) was used. P value $<0.05$ was considered statistically significant.

\section{Results and Discussion}

At the end of follow-up period, cumulative incidence of DN progression was $25.7 \%$ (of these $1.6 \%$ progressed from normoalbuminuria to microalbuminuria, $1.6 \%$ from normo- to proteinuria, $31.2 \%$ from microalbuminuria to proteinuria, $15.6 \%$ from microalbuminuria to ESRD and 50\% from proteinuria to ESRD). Cumulative incidence of major cardiovascular event was $22.6 \%$ and of all-cause mortality $24.7 \%$, respectively. Ascertained genotype frequencies were: CC 57\%, CG 39\%, GG 4\% for SNP Arg72Pro in TP53 and TT $40 \%$, TG 51\%, GG 9\% for SNP309 in MDM2.

To assess the eventual impact of both SNPs on studied end-points we used Kaplan-Meier analysis. Results of the analyses are shown in Table 2. Given the low number of GG genotypes for the TP53 Arg72Pro SNP, we applied a dominant model and found significant difference between CG+GG vs. CC genotypes for DN progression $(\mathrm{P}=0.046, \log$ rank test). $\mathrm{DN}$ progression was faster in carriers genotypes containing $\mathrm{G}$ allele (associated previously with higher risk of T2DM [10] ), i.e. genotypes GG and GC compared to CC homozygotes, Kaplan-Meier curves are shown in Figure 1. We did not find any significant difference between genotypes of MDM2 SNP for any of the end-points studied.

Protein p53 has been traditionally viewed as a tumour suppressor activated by genotoxic stress with subsequent cell cycle arrest and repair of the damage or induction of apoptosis if the extent of changes was too

\begin{tabular}{|c|c|c|c|c|c|}
\hline Parameter (unit) & normoalbuminuria $(\mathrm{n}=27)$ & microalbuminuria $(n=104)$ & proteinuria $(n=130)$ & $\operatorname{ESRD}(n=48)$ & $P$ \\
\hline Age (years) & $66[61-72]$ & $68[57-77]$ & $66[58-75]$ & $69[64-75]$ & 0.179 \\
\hline Duration of diabetes (years) & $14[10-22]$ & $8[5-14]$ & $14.5[8-20]$ & $17[12-22]$ & $<0.001$ \\
\hline $\mathrm{FPG}(\mathrm{mmol} / \mathrm{l})$ & $7.7[6.2-10.2]$ & $7.9[7.3-10.5]$ & $9.3[7.5-11.3]$ & $7.8[6.3-11]$ & 0.173 \\
\hline $\mathrm{HbA}_{1 \mathrm{C}}(\%)$ & $6.4[5.2-7.5]$ & $6.45[5.35-8.1]$ & $8.1[6-9.6]$ & $6.2[5.2-7.8]$ & 0.062 \\
\hline Total cholesterol (mmol/l) & $4.9[4.3-6.3]$ & $4.9[4.3-5.7]$ & $5.2[4.3-6.2]$ & $4.8[3.9-5.5]$ & 0.323 \\
\hline Triglycerides $(\mathrm{mmol} / \mathrm{l})$ & $1.88[1.32-2.97]$ & $2[1.32-2.71]$ & $2.15[1.52-3.52]$ & $2.11[1.62-3.11]$ & 0.027 \\
\hline Creatinin $(\mu \mathrm{mol} / \mathrm{l})$ & $90[83-110]$ & 114 [92 - 147] & 139 [104 - 175] & 420 [250 - 550] & $<0.001$ \\
\hline Proteinuria (g/24h) & $0.11[0.08-0.13]$ & $0.14[0.08-0.22]$ & $1.33[0.4-3.06]$ & - & $<0.001$ \\
\hline GFR $\left(\mathrm{ml} / \mathrm{min}\right.$ per $\left.1.73 \mathrm{~m}^{2}\right)$ & $1.62[1.32-2.3]$ & $1.1[0.7-1.6]$ & $0.88[0.71-1.38]$ & - & $<0.001$ \\
\hline UAE (mg/24h) & $10[8-18]$ & 50 [21 - 160] & $181[97-545]$ & - & $<0.001$ \\
\hline
\end{tabular}

Data are expressed as median [interquartile range]. Comparisons were made by Kruskal-Wallis ANOVA.

Abbreviations: ESRD, end-stage renal disease; FPG, fasting plasma glucose; GFR, glomerular filtration rate; UAE, urinary albumin excretion.

Table 1: Clinical characteristics of the study participants. 
Citation: Kuricova K, Pacal L, Dvorakova V, Kankova K (2014) Association of the Arg72Pro Polymorphism in p53 with Progression of Diabetic Nephropathy in T2DM Subjects. J Nephrol Ther 4: 153. doi:10.4172/2161-0959.1000153

Page 3 of 3

\begin{tabular}{|c|c|c|c|c|}
\hline Gene (rs number) & Genotype & \multicolumn{3}{|c|}{ P } \\
\hline & & DN progression & CVE & ACM \\
\hline TP53 (rs1042522) & CC vs.CG vs. GG & 0.05 & NS & NS \\
\hline & CC vs.CG + GG & 0.046 & NS & NS \\
\hline & CC + CG vs. GG & NS & NS & NS \\
\hline MDM2 (rs2279744) & TT vs. TG vs. GG & NS & NS & NS \\
\hline & TT vs. TG +. GG & NS & NS & NS \\
\hline & TT + TG vs. GG & NS & NS & NS \\
\hline
\end{tabular}

Comparisons were made by log-rank test.

Abbreviations: DN: Diabetic Nephropathy; CVE: Cardiovascular Event; ACM: AllCause Mortality.

Table 2: Time-to-event analysis of individual SNPs.

\section{Arg72Pro TP53 vs. progression of DN}

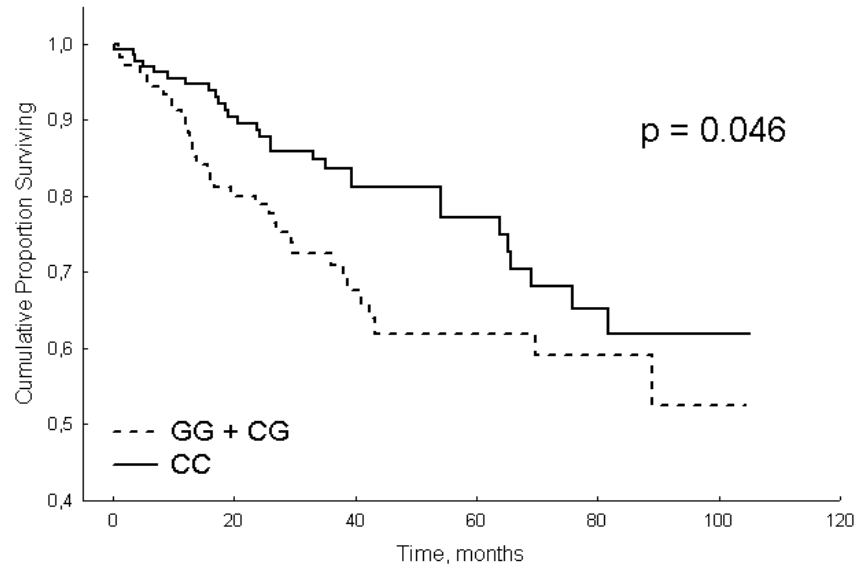

Figure 1: Kaplan-Meier curves for renal end-point for pooled genotypes CC vs. GG+GC DN, diabetic nephropathy.

large. However, in the last several years substantial evidence showed that p53 also has an important role in the regulation of metabolic pathways. This enabled a major shift of the Warburg effect paradigm and brought p53 into the focus in many other disciplines apart from oncology, e.g. diabetes and metabolic diseases. Hyperglycaemia has been shown to induce mobilization of p53 to the mitochondrial membrane with subsequent changes in mitochondrial membrane potential [18]. Hyperglycaemia also activates p53 in human endothelial cells [19]. Interestingly, oscillating glucose is more effective in p53 activation than constant high glucose [19] supporting the view of high glucose variability as an additional cellular stressor. Altogether, however, data elucidating the role of p53 in metabolic diseases like diabetes mellitus and its consequences are scarce so far. To our knowledge this is the first study exploring possible relationship between p53 and MDM2 SNPs and the progression of adverse effects of diabetes, namely diabetic nephropathy, cardiovascular morbidity and mortality.

\section{Conclusions}

In the current study we ascertained significant association of common polymorphism (specifically genotypes containing allele previously associated with higher risk of T2DM) with progression of diabetic nephropathy in subjects with T2DM. Elucidation of mutual contribution of TP53 genetic effect, long-term metabolic compensation and glycemic variability for progression of diabetic nephropathy definitely warrants further studies.

\section{Acknowledgements}

This work was supported by the European Regional Development Fund and the State Budget of the Czech Republic (RECAMO, CZ.1.05/2.1.00/03.0101).

\section{Conflict of Interest}

None declared

\section{References}

1. Vousden KH, Prives C (2009) Blinded by the Light: The Growing Complexity of p53. Cell 137: 413-431.

2. Vousden KH, Ryan KM (2009) p53 and metabolism. Nat Rev Cancer 9: 691-

3. Maddocks OD, Vousden KH (2011) Metabolic regulation by p53. J Mol Med (Berl) 89: 237-245

4. Bensaad K, Tsuruta A, Selak MA, Vidal MN, Nakano K, et al. (2006) TIGAR, a p53-inducible regulator of glycolysis and apoptosis. Cell 126: 107-120.

5. Rabbani N, Thornalley PJ (2011) Emerging role of thiamine therapy for prevention and treatment of early-stage diabetic nephropathy. Diabetes Obes Metab 13: 577-583.

6. Muller PA, Vousden KH (2013) p53 mutations in cancer. Nat Cell Biol 15: 2-8.

7. Dumont P, Leu JI, Della Pietra AC 3rd, George DL, Murphy M (2003) The codon 72 polymorphic variants of p53 have markedly different apoptotic potential. Nat Genet 33: 357-365.

8. Gaulton KJ, Willer CJ, Li Y, Scott LJ, Conneely KN, et al. (2008) Comprehensive association study of type 2 diabetes and related quantitative traits with 222 candidate genes. Diabetes 57: 3136-3144.

9. Minamino T, Orimo M, Shimizu I, Kunieda T, Yokoyama M, et al. (2009) A crucial role for adipose tissue p53 in the regulation of insulin resistance. Nat Med 15: 1082-1087.

10. Burgdorf KS, Grarup N, Justesen JM, Harder MN, Witte DR, et al. (2011) Studies of the association of Arg72Pro of tumor suppressor protein p53 with type 2 diabetes in a combined analysis of 55,521 Europeans. PLoS One 6: e15813.

11. Bonfigli AR, Sirolla C, Testa R, Cucchi M, Spazzafumo L, et al. (2013) The p53 codon 72 (Arg72Pro) polymorphism is associated with the degree of insulin resistance in type 2 diabetic subjects: a cross-sectional study. Acta Diabetol 50: 429-436.

12. Gloria-Bottini F, Banci M, Saccucci P, Nardi P, Scognamiglio M, et al. (2012) p53 codon 72 polymorphism and coronary artery disease: evidence of association with left ventricular ejection fraction. Am J Med Sci 343: 127-130.

13. Gloria-Bottini F, Banci M, Saccucci P, Neri A, Bottini E, et al. (2012) p53 codon 72 polymorphism and coronary artery disease: evidence of interaction with ACPâ,. Med Sci Monit 18: CR712-715.

14. Nag S, Qin J, Srivenugopal KS, Wang M, Zhang R (2013) The MDM2-p53 pathway revisited. J Biomed Res 27: 254-271.

15. Bond GL, Hu W, Levine A (2005) A single nucleotide polymorphism in the MDM2 gene: from a molecular and cellular explanation to clinical effect. Cancer Res 65: 5481-5484.

16. Zauberman A, Flusberg D, Haupt Y, Barak Y, Oren M (1995) A functional p53-responsive intronic promoter is contained within the human mdm2 gene. Nucleic Acids Res 23: 2584-2592.

17. Ohmiya N, Taguchi A, Mabuchi N, Itoh A, Hirooka Y, et al. (2006) MDM2 promoter polymorphism is associated with both an increased susceptibility to gastric carcinoma and poor prognosis. J Clin Oncol 24: 4434-4440.

18. Ortega-Camarillo C, Guzmán-Grenfell AM, García-Macedo R, Rosales-Torres AM, Avalos-Rodríguez A, et al. (2006) Hyperglycemia induces apoptosis and p53 mobilization to mitochondria in RINm5F cells. Mol Cell Biochem 281: 163 171.

19. Schisano B, Tripathi G, McGee K, McTernan PG, Ceriello A (2011) Glucose oscillations, more than constant high glucose, induce p53 activation and a metabolic memory in human endothelial cells. Diabetologia 54: 1219-1226. 